\title{
NEW LOWER BOUNDS FOR THE HILBERT NUMBERS USING REVERSIBLE CENTERS
}

\author{
R. PROHENS AND J. TORREGROSA
}

\begin{abstract}
In this paper we provide the best lower bounds, that are known up to now, for the Hilbert numbers of polynomial vector fields of degree $N, H(N)$, for small values of $N$. These limit cycles appear bifurcating from new symmetric Darboux reversible centers with very high simultaneous cyclicity. The considered systems have, at least, three centers, one on the reversibility straight line and two symmetric about it. More concretely, the limit cycles are in a three nests configuration and the total number of limit cycles is at least $2 n+m$, for some values of $n$ and $m$. The new lower bounds are obtained using simultaneous degenerate Hopf bifurcations. In particular, $H(4) \geq 28, H(5) \geq 37, H(6) \geq 53, H(7) \geq 74$, $H(8) \geq 96, H(9) \geq 120$, and $H(10) \geq 142$.
\end{abstract}

\section{INTRODUCTION}

We consider two-dimensional differential systems

$$
\dot{x}=P(x, y), \quad \dot{y}=Q(x, y),
$$

in which $P$ and $Q$ are polynomials of degree $N$. The maximum possible number, $H(N)$, of limit cycles of system (1) is known as the Hilbert number. As usual we define limit cycle as every isolated periodic solution.

As it follows from former definition, the Hilbert number refers to the total amount of limit cycles that system (1) exhibits and, in this sense, it is a global concept. For instance, Shi (see [24]) and Chen and Wang (see [5]) proved that $H(2) \geq 4$. For cubics, Li, Liu, and Yang (see [18]) and Li and Liu (see [19]) proved that $H(3) \geq 13$. In our work we prove, among other new best lower bounds for the Hilbert numbers, that $H(4) \geq 28$.

The aim of this work is to obtain new lower bounds values for $H(N)$. This goal is attained by simultaneously perturbing some reversible centers. We proceed studying simultaneous degenerate Hopf bifurcations of reversible centers and, to overcome heavy computations, we use an efficient way (parallelization method) for the Lyapunov quantities calculation.

This work strongly relies on the results of Christopher, [7], and Han, [12] and [13]. The idea is to estimate the generic cyclicity of a family of simultaneous centers from the series expansion of the Lyapunov quantities at a point on the center variety. In particular, if the first $r$ linear terms of the Lyapunov quantities are independent, then the cyclicity is bigger or equal than $r$, if we consider also the trace as another independent parameter. The germ of this linearization idea can be glimpsed at the work of Chicone and Jacobs, see [6], where, for higher order limit cycles bifurcations,

Date: January 10, 2018.

2010 Mathematics Subject Classification. Primary 34C07, Secondary: 37G15, 34C23, 34C25.

Key words and phrases. Polynomial differential equation; Limit cycles; Bifurcation and number of periodic orbits; 16th Hilbert number. 
the Lyapunov quantities are obtained from the Taylor series expansion of the perturbation parameters. We want to remark that, in the paper [7], Christopher studies this symmetric simultaneous bifurcation, only up to first order perturbation, of a quartic system obtaining 22 limit cycles in two symmetric nests of 6 cycles and one nest of 10. For short, we say that these limit cycles are in configuration $\langle 6,10,6\rangle$. In Section 3 we give the precise definition of the configuration concept and we describe de complete bifurcation mechanism.

The present work can be considered as a continuation of the Christopher's one since, to obtain limit cycles, we use the same mechanism to study symmetric simultaneous bifurcation of limit cycles but using higher order perturbation for the same center of degree 4 and linear order for a new Darboux centers of bigger degrees. Our main results are the following.

Theorem 1. $H(4) \geq 28, H(5) \geq 37, H(6) \geq 53, H(7) \geq 74, H(8) \geq 96$, $H(9) \geq 120$, and $H(10) \geq 142$. Moreover, the configurations of limit cycles for $N=$ $4, \ldots, 10$, are $\langle 8,12,8\rangle,\langle 11,15,11\rangle,\langle 16,21,16\rangle,\langle 23,28,23\rangle,\langle 30,36,30\rangle,\langle 38,44,38\rangle$, and $\langle 45,52,45\rangle$, respectively.

Corollary 2. (a) $H(13) \geq 212, H(17) \geq 384, H(21) \geq 568, H(31) \geq 1184$, $H(35) \geq 1536, H(39) \geq 1920$, and $H(43) \geq 2272$.

(b) For each pair $\left(N_{0}, K_{0}\right) \in\{(4,28),(5,37),(6,53),(7,74),(8,96),(9,120),(10,142)\}$, we have that

$$
H(N) \geq \frac{K_{0}}{\left(N_{0}+1\right)^{2}} N^{2}
$$

when $N=2^{k}\left(N_{0}+1\right)-1$, for $k \in \mathbb{N}$.

The linear computational approach to estimate lower bounds for the cyclicity of centers has, at least, two advantages. The first one is computational, and it is based on the fact that, by shortening the length of the expressions to be manipulated, more fast computations can be performed. The second one has to do with the functional independence argument of the Lyapunov quantities, since this argumentation is replaced by an study, in most of the cases, on the independence of their linear parts.

Nevertheless, sometimes, linear terms of the Lyapunov quantities are not independent and, for studying the simultaneous bifurcation, we must take into account higher order terms. This disadvantage was considered by Christopher and, to overcome the setback that the computations are no longer linear and become unmanageable, under some generic assumptions, something specific can be proved, see [7, Th.3.1].

The improvement for $N=4$, with respect to Christopher's work, is that we obtain 28 limit cycles in two nests of 8 cycles and one nest of 12 , i.e. in a $\langle 8,12,8\rangle$ configuration. The key point in obtaining this new best estimation of $H(4)$ is the use of fifth order, instead of linear order, approximation of the Lyapunov quantities. It is also worth mentioning that, despite having tried to use this technique in the cubic case, we have not been able to improve the current lower bound of $H(3)$. See, respectively, Sections 5 and 4.

Concerning the parallelization method, roughly speaking, it is as follows. We, one-by-one, compute the linear part of a fixed number of Lyapunov quantities of simpler differential equations having only one perturbation monomial. Then, by taking the summation of all the results, we obtain the corresponding linear part of the Lyapunov quantity of the complete differential equation. If we proceed in this 
way it can be checked that the computations in each simpler equation are shorter in size and time. See [20] for more details.

In our paper, we follow former approach and latter technique applied to families of polynomial reversible symmetric differential systems (1) having two centers outside the symmetry line, which will be the $x$-axis, plus one more extra center at the origin. See Figure 1. Then, we force the existence of two symmetric weak foci of order $n$

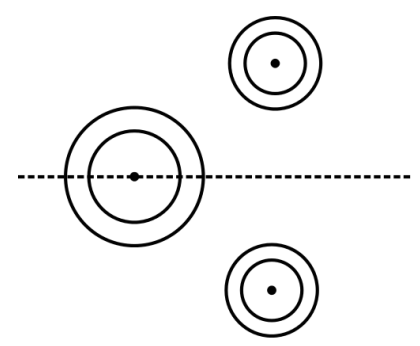

FiguRE 1. Schematic phase portrait of the polynomial reversible symmetric differential systems (1) having one center at the origin plus two more symmetric centers.

where the symmetric centers were located, out off the $x$-axis. We aim to create $n$ limit cycles, by using only reversible perturbations, around each one of them; in this way, $2 n$ simultaneous limit cycles will be achieved. After that, by perturbing again the system, but without the reversibility property, we can generate $m$ more limit cycles surrounding a weak focus of order $m$ at the origin. Summing up, the total number of limit cycles we have generated is $2 n+m$, in configuration $\langle n, m, n\rangle$.

The proof of Theorem 1 is done in Sections 5,6 and 7, while Corollary 2 is proved in Section 8. We note that the supplied lower bounds for the Hilbert numbers, $H(N)$, for $N=4, \ldots, 10$, in Theorem 1 significantly improve the previous best known Hilbert numbers. See for instance $[14,15,20]$. We also give a a new general lower bound for the Hilbert numbers, $H(N)$. See Corollary 2.

Even taking into account that the parallelization method has more facilities than using other ways to proceed in finding new better lower bounds of $H(N)$, some obstacles are found in to go ahead for values of $N$ higher than those considered. Next, we comment some of them. The first obstruction concerns the computer memory. In spite off the usage of linear approximations, the computations of Lyapunov quantities for higher values of $N$ requires, increasing $N$, more and more memory. Another arising problem is the necessity to get reversible differential systems providing good seeds for bifurcating the required number of limit cycles. Finally, another problem, for our computational approach, is how to obtain good first integrals in such a way that, when moving to complex variables, the coefficients in the differential equations are all rational numbers.

This paper is structured as follows. In Section 2 we recall, not only some of the known best estimates of lower bounds for the Hilbert numbers, but also the corresponding known best estimations of lower bounds for the cyclicity of non degenerated monodromic equilibrium points. In Section 3 we describe the methodology used to get the main results. In Section 4 we provide a detailed description of the method applied to a cubic vector field, getting a characterization of all cubic systems having a reversible center with two extra symmetric centers out of the symmetry axis. In Section 5, we study some reversible quartic systems and, by considering fifth order 
terms in the Lyapunov quantities Taylor series, and we get the new best lower bound $H(4) \geq 28$. Section 6 is devoted to get some families of reversible polynomial vector fields of degrees $5, \ldots, 9$ with a rational first integral having simultaneous bifurcation of limit cycles. In this section we present, among other lower bounds for the Hilbert numbers, the new best lower bounds $H(5) \geq 37$ and $H(6) \geq 53$. In Section 7 we study some Darboux reversible centers having a curve of equilibria. In this section we obtain the new best lower bounds $H(7) \geq 74, H(8) \geq 96, H(9) \geq 120$ and $H(10) \geq 142$. Finally, in Section 8 we prove Corollary 2.

\section{Previous estimations for $M(N)$ and $H(N)$}

Before to start with the global concept of the Hilbert number, $H(N)$, a local concept concerning the bifurcation of small limit cycles around a specific equilibrium point is also considered. This is the case for the cyclicity of an equilibrium point.

Roughly speaking, a monodromic equilibrium point for a family of vectors fields in (1) has finite cyclicity $M(N)$ if, in Hausdorff distance, the maximum number of limit cycles is $M(N)$, and they appear in a close neighbourhood of the equilibrium point, when the perturbation tends to zero whereas the neighbourhood shrinks to the equilibrium point. From previous definitions it is easy to conclude that $M(N) \leq$ $H(N)$, for all $N$.

In this section we present the known best estimations, up to now, of $M(N)$ and $H(N)$. As we prove in this work, some of them will be improved from our results.

A classical bifurcation mechanism to study the existence of periodic orbits around an equilibrium point of monodromic type is the non-degenerate Hopf bifurcation method. This mechanism studies the limit cycles that emerge from an equilibrium point of center-focus type when, by changing the sign of the trace in a suitable way, its stability changes from stable to unstable or vice-versa. The non-degenerate Hopf bifurcation method can be generalized, by computing the Lyapunov quantities, to more degenerate critical points. This generalization is known as the degenerated Hopf bifurcation method and it is used to give estimations of $M(N)$, see [1], for instance. Besides former reference, see also [13, 30] for more results on bifurcation theory of limit cycles. Among these specialized references, a general approach to qualitative theory of differential equation can be found in [9].

In the last decades some improvements of the lower bounds of $H(N)$ have been achieved by using better estimates of $M(N)$. In fact, this is the case for some special small values of $N$. At this point it is worth to mention that only for quadratic polynomial vector fields we know the exact value of it, that is $M(2)=3$. This fact was proved by Bautin in [2]. Concerning $M(N)$ for $N \geq 3$, up to now, only lower bounds for these numbers has been obtained, as detailed below.

In finding lower bounds of $H(N)$ it is worth mentioning that one of the most used techniques to create limit cycles is by perturbing $Z_{q}$-equivariant polynomial Hamiltonian vector fields. Frequently, this technique is combined with other procedures such as: Hopf bifurcation method, homoclinic and heteroclinic bifurcation methods, Abelian integrals and bifurcation from infinity, ..., see $[9,13,23]$.

The known best result on $M(3)$ was obtained by Żołądek in [31], getting $M(3) \geq$ 11. This work has been amended in [33] although the same bound for $M(3)$ is maintained. The proof was made, after a cubic perturbation of a cubic differential equation having a rational first integral, by bounding the zeros of the displacement 
map. This displacement map, in first approximation, is given by a linear PoincaréPontriaguin integral which is expressed in terms of twelve Abelian or Melnikov type integrals (first order Melnikov integrals) generating the aforementioned eleven limit cycles. Linked with the first paper of Żoładek, in 2005, Christopher in [7], among other results, confirmed the lower bound for cubic polynomial vector fields, that is $M(3) \geq 11$. He uses a family of cubic systems, $C_{31}^{(12)}$ according Żołądek's more recent classification [32], having also a rational first integral and showing that the linear part of the first Lyapunov quantities have maximal rank 11. In [4], Bondar and Sadovski also proved, with the same technique but with a different Darboux center, that $M(3) \geq 11$.

In the case $N=4$, that is on quartics systems, in [11], Giné proved that $M(4) \geq$ 21 , by studying small limit cycles bifurcating from an elementary center-focus type equilibrium point. In the case $N=5$, i.e. concerning lower bounds of $M(5)$, the known best estimation is the one given by Giné in [11], where $M(5) \geq 26$ was proved. These limit cycles also bifurcate from an elementary center-focus type equilibrium point at the origin. In this paper, Giné conjectured that the number of functionally independent focal values, i.e. the minimum number of ideal generators of the ideal generated by the focal values, of system (1) with an elementary center-focus type equilibrium point at the origin is $M(N)=N^{2}+3 N-7$. We want to point out that, regarding Giné's conjecture, we would have $M(4)=21$ and $M(5)=33$.

In the cases $N=6, \ldots, 13$, Liang and Torregrosa in [20] proved that the cyclicity of some holomorphic Darboux center for system (1) is, at least, $N^{2}+N-2$, for $4 \leq N \leq 13$. Hence, we have the lower bound $H(N) \geq M(N) \geq N^{2}+N-2$, for $4 \leq N \leq 13$. We point out that in that work, the authors say that the best center candidate to produce high cyclicity is of Darboux type. One consequence of their paper is that, by one hand, this result gives the highest known up to now lower bound of $M(6), M(7), \ldots, M(13)$, and that these numbers are smaller than those conjectured before. In particular, for $N=6,8,10$ are also the best lower bound for Hilbert numbers, i.e. $H(6) \geq 40, H(8) \geq 70$, and $H(10) \geq 108$.

A lower bound of $M(N)$, for all $N$, was given by Movasati, see [22, Cor 4.1]. In this paper it is proved that, the cyclicity of holomorphic centers perturbing with polynomial degree $N$ systems is not less than $M(N) \geq N^{2}-2$. In regards to upper bounds of $M(N)$, Mardesic, in [21], proved that an upper bound for the cyclicity of a period annulus around an equilibrium point of center-focus type, given by a Hamiltonian polynomial of degree $N$ differential equation, is $M(N) \leq\left(N^{4}+N^{2}-\right.$ $2) / 2$. This bound was obtained as an upper bound of the number of zeros of the corresponding Abelian integral.

Next we summarize some of the recent progress in finding lower bounds of $H(N)$.

On $H(2)$, Shi (see [24]) and Chen and Wang (see [5]) proved that $H(2) \geq 4$. They obtained these four limit cycles in two nests, one with 3 and another with 1.

On $H(3)$, in 2009, Li, Liu, and Yang (see [18]), by counting the number of zeros of some Abelian integrals, demonstrated that an specific planar cubic system has $H(3) \geq 13$. Later, Li and Liu (see [19]) also proved that $H(3) \geq 13$, using $Z_{2}$-equivariant cubic perturbations, Hopf bifurcation and changing the stability of infinity. These 13 limit cycles appear in two nests of 6 and one coming from infinity.

About $H(4)$, Christopher, [7] in 2005, provided a quartic system with $H(4) \geq 22$. As we have mentioned before, he used the linear parts of the Lyapunov quantities 
for center bifurcations with symmetries, the limit cycles appear in two nests of 6 and one nest of 10. Recently, in 2011, Johnson constructed (see [15]) a quartic system that has 24 limit cycles, using Lyapunov quantities, in four nests of 6 . Moreover, the existence of 2 more, having each one inside two of these nests, is showed numerically. Then, $H(4) \geq 26$.

In the study of $H(5)$, in 2008, Wu, Gao, and Han in [28] found that $H(5) \geq$ 28 , in four different configurations, by using the perturbation of a $Z_{2}$-equivariant symmetric quintic Hamiltonian system. Later, in 2010, Wu, Wang, and Tian, in [29] proved also $H(5) \geq 28$ with a $Z_{4}$-equivariant symmetric quintic vector field, by using the Hopf and polycycle bifurcation methods. Also in 2010, Johnson and Tucker, in [17], studied the limit cycle bifurcation of a $Z_{2}$-equivariant quintic planar Hamiltonian vector field under $Z_{2}$-equivariant quintic perturbation and, by computer aided approach, proved that $H(5) \geq 27$. In 2015, Sun and Han in [25] also proved that $H(5) \geq 28$ using $Z_{4}$-equivariant perturbations.

Concerning $H(6)$, in 2005, Wang and $Y u$, showed that $H(6) \geq 35$, by using global and local bifurcations, see [26]. In this paper the authors perturb a fifth-degree $Z_{2}$-equivariant symmetric Hamiltonian system by adding a sixth-degree polynomial system. More recently, in 2015, Liang and Torregrosa, by computing the required independent linear parts of the Lyapunov quantities, proved that $H(6) \geq M(6) \geq$ 40 , see $[20]$.

On $H(N)$, for $N \geq 7$, it is worth mentioning the relevant paper of Han and Li [14] in 2012. The results in this work improved almost all existing lower bounds of $H(N)$ for such values of $N$, up to that moment. More concretely, they proved that $H(7) \geq 65, H(9) \geq 98, H(11) \geq 153, H(12) \geq 157, \ldots$ In 2010, Johnson and Tucker in [16] proved that $H(7) \geq 53$, perturbing a $Z_{2}$-equivariant planar Hamiltonian vector field of degree 7 . The proof follows a computer aided approach. For $N=11$, before the paper of $\mathrm{Han}$ and $\mathrm{Li}$, the best one was the one given by Wang and $\mathrm{Yu}$ in 2006 , in [27], proving that $H(11) \geq 121$ using a $Z_{12}$-equivariant vector field. With respect to $H(8)$, until 2012 the best lower bound for this Hilbert number was the one given by $\mathrm{Han}$ and $\mathrm{Li}$, as $H(8) \geq 67$. Nevertheless, in 2015, Liang and Torregrosa, in [20], obtained a new better one, that is $H(8) \geq M(8) \geq 70$. In this paper it was also proved that $H(10) \geq M(10) \geq 108$.

Concerning the rate of grow of $H(N)$ as $N$ increases, one of the first results in finding a general lower bound we want to point out, is the work of Christopher and Lloyd in 1995, see [8], where they introduced a recurrence mechanism to provide a lower bound for $H(N)$ of type $K N^{2} \log N$. Recently, in 2012, Han and Li improved this mechanism by providing a better lower bound of the same kind, see [14]. Concretely, for $N$ big enough, they showed that $H(N)$ grows at least as fast as $(N+2)^{2} \log (N+2) /(2 \log 2)$.

\section{The BIFURCATION MECHANISM}

The bifurcation mechanism, in short, is as follows. First, we consider a reversible system (symmetric with respect to the $x$-axis) having a center at the origin and two more at two symmetric points $\left(x_{c}, \pm y_{c}\right)$, with $x_{c}^{2}+y_{c}^{2} \neq 0$. Second, we consider a first perturbation obtaining $n$ (simultaneously) hyperbolic limit cycles in two nests while the origin remains as a center. Finally, we consider a second perturbation of the system giving us $m$ limit cycles, around this third nest, increasing the total 
number of limit cycles obtained. Then, we will say that the $2 \times n+m$ limit cycles appear in configuration $\langle n, m, n\rangle$.

The limit cycles appear using degenerated Hopf bifurcations of some order $k$ and all the unperturbed polynomial systems have rational first integrals. Concerning the Lyapunov quantities computation we use linear or higher order developments of Lyapunov quantities, as in the works of Christopher ([7]) and Han ([12]). In this paper, reversible differential systems in the $(x, y)$-plane means invariant under the change of variables $(x,-y,-t) \rightarrow(x, y, t)$. Moreover, all the centers are non degenerate.

As all the computations are quite hard to do and the obtained expressions are too big to be written here, we will describe only the bifurcation mechanism more in detail. This bifurcation scheme is the same followed by Christopher (see also [7]) but we use higher instead of first order. In some simple cases and for lower degrees we will also show which are the Taylor series of the Lyapunov quantities.

3.1. Degenerated Hopf bifurcation. An analytic system with a non-degenerate equilibrium point of center-focus type can be transformed to the system

$$
\begin{aligned}
x^{\prime} & =\lambda x-y+f(x, y), \\
y^{\prime} & =x+\lambda y+g(x, y),
\end{aligned}
$$

where $f$ and $g$ are analytic functions with lower order terms of degree 2 in $x, y$, being $\lambda$ a real parameter and with the equilibrium point located at the origin.

When $\lambda=0$ we have a weak focus or a center at the origin. The problem to distinguish its stability is the well-known center-focus problem. There are some classical approaches to solve it, see [1] for instance. Here we will use the Andronov-Poincaré method, that is the computation of the obstruction conditions for its integrability, but using complex notation. Hence, following the approach of [24], system (2) writes, in complex coordinates $(z=x+i y)$, as

$$
z^{\prime}=(\lambda+i) z+F_{2}(z, \bar{z})+F_{3}(z, \bar{z})+\cdots,
$$

where $F_{j}$ are homogeneous polynomials of degree $j$ in $z, \bar{z}$.

Now, if $\lambda=0$, we look for the existence of a formal first integral of the form

$$
H(z, \bar{z})=z \bar{z}+H_{3}(z, \bar{z})+H_{4}(z, \bar{z})+\cdots,
$$

with $H_{j}$ homogeneous polynomials of degree $j$ in $z, \bar{z}$. This can be done imposing that the level curves of $H$ contain solutions of equation (3). Straightforward computations show that the coefficients of $H_{3}$ can be uniquely determined in terms of the coefficients of $F_{2}$, by solving the diagonal linear system of equations obtained vanishing all the coefficients in $z, \bar{z}$ of the homogeneous polynomial of degree 3

$$
\bar{z} F_{2}+z \bar{F}_{2}+i z \frac{\partial H_{3}}{\partial z}-i \bar{z} \frac{\partial H_{3}}{\partial \bar{z}} .
$$

We can not follow exactly in the same way, to fix the coefficients of $H_{4}$, because the linear system of equations obtained vanishing the coefficients of the homogeneous polynomial of degree 4 ,

$$
\bar{z} F_{3}+z \bar{F}_{3}+i z \frac{\partial H_{4}}{\partial z}-i \bar{z} \frac{\partial H_{4}}{\partial \bar{z}}+\frac{\partial H_{3}}{\partial z} F_{2}+\frac{\partial H_{3}}{\partial \bar{z}} \bar{F}_{2} \equiv 0,
$$

has not maximum rank five. This obstruction can be removed by adding an extra term, $L_{1} z^{2} \bar{z}^{2}$ for example, in the above homogeneous identity. This constant $L_{1}$ 
is known as the first Lyapunov quantity. Clearly, $H$ is a Lyapunov function when $L_{1} \neq 0$ and gets the stability of the origin. When $L_{1}=0$, we need to compute higher degree terms of the function $H$ for distinguishing when the equilibrium point is a center or a focus. This constant $L_{1}$ can be thought as the first obstruction for (3) being integrable. In the recursive procedure to get $H_{j}$, for $j=3, \ldots$, it can be proved that all the linear systems giving the coefficients of $H_{2 k+1}, k=1,2, \ldots$, have maximum rank. Consequently $H_{2 k+1}$ can be uniquely determined. But in each even step, i.e. to determine $H_{2 k+2}, k=1,2, \ldots$, we need to add a correction term of the form $L_{k} z^{k+1} \bar{z}^{k+1}$. These quantities $L_{k}$ are the well known Lyapunov quantities associated to equation (3). Clearly, the first non-vanishing Lyapunov quantity, $L_{k}$, gives the stability of the origin. In this case, as usual, we say that the origin is a weak focus of order $k$. We would remark that the main computational difficulties to obtain the Lyapunov quantities, applying this procedure or in general other methods, are only because of their huge expressions.

For polynomial systems, the Lyapunov quantities can be used, besides to determine the stability of the origin and the center conditions of system (2), to obtain periodic orbits from the origin. For example when $L_{1}<0$ and $\lambda=0$ the origin is stable and for $\lambda>0$, small enough, the stability of the origin changes to unstable and an small stable hyperbolic periodic orbit bifurcates from the origin. This is the well-known Hopf bifurcation. An analogous procedure can be used to create $k$ limit cycles from a weak focus of order $k$ by a degenerate Hopf bifurcation. These limit cycles are also known as small limit cycles. See [3], for instance.

Now we will describe how the small limit cycles appear in a degenerate Hopf bifurcation. Suppose that system (2) is a polynomial system, that we call system $S$, having, without loss of generality, an stable weak focus of order $k$ at the origin. Then, $\lambda=0$ and the Lyapunov quantities satisfy $L_{1}=L_{2}=\cdots=L_{k-1}=0$, $L_{k}<0$. Let $\Gamma$ be a level curve of $H$ which is sufficiently near the origin that the flow is inward across it. Now we perturb system $S$ in such a way that the perturbed system, we call it system $S_{1}$, has $\lambda=L_{1}=L_{2}=\cdots=L_{k-2}=0, L_{k-1}>0$. The origin is thus unstable. If $S_{1}$ is sufficiently near $S$, the flow remains inward across $\Gamma$ and, if $H^{1}$ is the Lyapunov function corresponding to $S_{1}$, there exists a level curve $\Gamma_{1}$ of $H^{1}$ inside $\Gamma$ and sufficiently near the origin that the flow is outward across $\Gamma_{1}$. By the Poincaré-Bendixson Theorem, there is a limit cycle between $\Gamma$ and $\Gamma_{1}$. The next step is to take a perturbation, $S_{2}$, of system $S_{1}$ so that $L_{1}=\cdots=L_{k-3}=0$ and such that $L_{k-2}<0$. In this case, if the perturbation is sufficiently small, the flow remains inward across $\Gamma$ and outward across $\Gamma_{1}$. Hence, $S_{2}$ has a limit cycle between $\Gamma$ and $\Gamma_{1}$ and, since the origin is stable for $S_{2}$, there is also a limit cycle inside $\Gamma_{1}$. Proceeding in this way, $k$ limit cycles can be generated. The necessary conditions (denoting $L_{0}=\lambda$ ) are

$$
L_{j} L_{j+1}<0, \quad\left|L_{j}\right| \ll\left|L_{j+1}\right|, \quad j=0, \ldots, k-1 .
$$

When we study the degenerate Hopf bifurcation in a family of polynomial systems (2) of fixed degree, there is a classical problem: "the existence of parameters ensuring the independence of the Lyapunov quantities to get exactly $k$ periodic orbits near a weak focus of order $k$ ". Another related question is "which is the maximum weak focus order in a given family". Both questions concern to Hilbert's 16th problem, providing lower bounds for the Hilbert number, $H(N)$. A way to tackle this problem is obtaining polynomial families having a weak focus of the highest order that unfold 
this number of periodic orbits. We recall that, for analytical perturbations, always a weak focus of order $k$ unfolds $k$ hyperbolic limit cycles, see [23].

3.2. Cyclicity of centers. In this paper we select polynomial center families of degree $N$ having cyclicity at least $k$. We recall that the cyclicity at an equilibrium point can be defined as the maximum number of isolated periodic orbits bifurcating from it. In our approach this is equivalent to say that, in the parameters space, near the origin and after perturbing the center with polynomials also of degree $N$, there will be a curve of weak foci of order $k$ passing though the origin and unfolding $k$ hyperbolic periodic orbits. We remark that, in the parameters space, the origin corresponds to the case in which the system, not perturbed, has a center. As we can always obtain a limit cycle with the trace parameter, $\lambda$, we will restrict our analysis to the class of zero trace perturbations. That is, to the perturbations whose lower degree terms are of degree two. As we have mentioned before, to get the existence of these curves in the parameters space and the corresponding unfolding from the Taylor developments, we follow the ideas of Christopher, [7], and Han, [12]. The existence of a curve of weak focus is obtained studying the intersection of the Taylor developments of the varieties $L_{j}=0$, for $j=1, \ldots, k-1$ ensuring that along it $L_{k}$ is non-vanishing. The unfolding of $k$ limit cycles (adding the trace parameter) and their hyperbolicity is obtained from the transversality of the intersection of the varieties. Clearly, using the implicit function theorem, if the matrix defined by the linear terms of $L_{1}, \ldots, L_{k}$ have rank $k$ we have (adding $\lambda$ ) at least $k$ hyperbolic limit cycles bifurcating from the center point.

Consider the equation

$$
z^{\prime}=i z+F_{c}(z, \bar{z})
$$

in complex variables $(z=x+i y)$, with $F_{c}$ a polynomial starting with second order terms, having a center at the origin. The Lyapunov quantities, $L_{j}$, associated with equation

$$
z^{\prime}=i z+F_{c}(z, \bar{z})+F(z, \bar{z}),
$$

where $F$ is a polynomial perturbation, also starting with second order terms in $z, \bar{z}$, can be written, using its Taylor series in the parameters space, as

$$
L_{j}=L_{j}^{(1)}+L_{j}^{(2)}+L_{j}^{(3)}+\cdots,
$$

where $L_{j}^{(\ell)}$ are homogeneous polynomials of degree $\ell$ in the coefficients of $F$.

All the computations in this paper are done adapting the algorithm described above, see also [20], for obtaining directly the Taylor series of $L_{j}$ up to order $\ell$, generally $\ell=1$, and in some cases $\ell=2$ or higher, at the selected center.

3.3. Simultaneous Hopf bifurcations. The use of previous described center bifurcation method, combined with symmetry techniques (reversible systems), is a useful tool in obtaining lower bounds for the number of limit cycles in polynomial systems, see [8]. We use this tool, with two perturbations, to obtain limit cycles appearing simultaneously in three nests of limit cycles, two symmetric out of the symmetry line and one on it. We are going to present the idea of combining these two perturbations to generate limit cycles. 
Let $f$ and $g$ be polynomials in $x, y$. Consider a polynomial reversible system with an equilibrium point of center type at the origin

$$
\begin{aligned}
& x^{\prime}=-y+y f_{c}\left(x, y^{2}\right), \\
& y^{\prime}=x+g_{c}\left(x, y^{2}\right),
\end{aligned}
$$

having two more symmetric equilibrium points, $\left(x_{c}, \pm y_{c}\right)$, of center type out of the symmetry line. This configuration is depicted in Figure 1. We consider a general perturbation of degree $N$ of system (7) in two independent steps.

First, considering that we want to preserve the center at the origin, we take a perturbation of system (7) keeping the reversibility symmetry at the origin,

$$
\begin{aligned}
& x^{\prime}=-y+y f_{c}\left(x, y^{2}\right)+\sum_{k+2 \ell+1=1}^{N} f_{k, 2 \ell+1} x^{k} y^{2 \ell+1}, \\
& y^{\prime}=x+g_{c}\left(x, y^{2}\right)+\sum_{k+2 \ell=1}^{N} f_{k, 2 \ell} x^{k} y^{2 \ell} .
\end{aligned}
$$

We observe that is not restrictive to assume that the origin remains as a critical point. Then, we translate system (8) in order that the center $\left(x_{c}, y_{c}\right)$ moves to the origin. Additionally, we restrict the perturbation parameters $f_{k, \ell}$ such that the perturbation terms in the translated system start with terms of degree 2 . Then, the method of small limit cycles generation around the origin, described in the first part of this section, applies. Let $n$ be the maximum number of limit cycles obtained, around the origin, with this technique. Then, we have $n$ small limit cycles, by a simultaneous degenerated Hopf bifurcation, surrounding each one of the equilibrium points $\left(x_{c}, \pm y_{c}\right)$.

We remark that the origin of system (8) remains a center when the $2 n$ limit cycles have been created. Clearly, with this method we can obtain, at most, as limit cycles as the number of free parameters, that is $2 n \leq(N+5)(N-2) / 2$. In the next sections it is shown that in our best results never reach this number but, nevertheless, we are close to it.

The second step is to consider a perturbation that completely breaks the symmetry at the origin,

$$
\begin{aligned}
& x^{\prime}=-y+y f_{c}\left(x, y^{2}\right)+\sum_{k+2 \ell+1=2}^{N} e_{k, 2 \ell+1} x^{k} y^{2 \ell+1}, \\
& y^{\prime}=x+g_{c}\left(x, y^{2}\right)+\sum_{k+2 \ell=2}^{N} e_{k, 2 \ell} x^{k} y^{2 \ell} .
\end{aligned}
$$

Let $m$ be the maximum number of small limit cycles obtained using, once again, the previous described technique to study the degenerate Hopf bifurcation, but now applied to system (9). As above, we can obtain, at most, as many limit cycles as the number of free parameters; that is, $m \leq N(N+2) / 2$.

Finally, from the above arguments, and since the perturbation parameters in systems (8) and (9) are independent, we have that the $2 n$ small limit cycles surrounding the equilibria $\left(x_{c}, \pm y_{c}\right)$ are hyperbolic and remain after the other perturbation, i.e. they remain while $m$ limit cycles emerging from the origin. This method is used in detail, in the next section, for a cubic system. 
Considering all together, at least $2 n+m$ small limit cycles has been obtained; $m$ small limit cycles surrounding the origin and $n$ small limit cycles surrounding each one of the two symmetric equilibrium points. We will say that the $2 \times n+m$ limit cycles are in configuration $\langle n, m, n\rangle$.

\section{REVERSIBle CUBIC CENTERS With TWO EXTRA SYMMETRIC CENTERS OUT OF THE SYMMETRY LINE}

The aim of this section is to present the method introduced in Section 3, of simultaneous occurrence of limit cycles via a degenerate Hopf bifurcation, through a particular case. We introduce this method by studying some cubic reversible family of centers. We want to remark that using other families of cubic centers and other bifurcation techniques, more limit cycles can be obtained, see [18, 19].

First, see Proposition 3, we classify all reversible cubic systems having a nondegenerate equilibrium point of center type at the origin plus two more non-degenerate centers out of the reversibility symmetry line. Clearly, up to an affine change of variables and a time rescaling if necessary, it is not restrictive to assume that the symmetry axis is the straight line $y=0$. Hence, the reversible cubic centers family, having two extra symmetric centers out of the symmetry line, that we consider write as

$$
\begin{aligned}
& x^{\prime}=-y+a_{11} x y+a_{21} x^{2} y+a_{03} y^{3}, \\
& y^{\prime}=x+b_{20} x^{2}+b_{02} y^{2}+b_{30} x^{3}+b_{12} x y^{2} .
\end{aligned}
$$

Second, see Proposition 5, following the procedure described in the previous section, we study the simultaneous degenerate Hopf bifurcation of limit cycles in configuration $\langle 1,7,1\rangle$ for family (10).

As we will see in the proof we can not provide better examples using this bifurcation technique for cubic vector fields. We recall that the family of cubic systems can exhibit up to 13 limit cycles, as it is proved in $[18,19]$.

We finish this section showing some systems of family (10), with different phase portraits, exhibiting the same configuration $\langle 1,7,1\rangle$ of limit cycles.

Proposition 3. After a rescaling, if necessary, the reversible cubic system (10) has three non-degenerate centers, one at the origin and two at $\left(x_{c}, \pm y_{c}\right)$ with $x_{c}^{2}+y_{c}^{2} \neq 0$, if and only if, the determinant of the Jacobian matrix of system (10) at $\left(x_{c}, y_{c}\right), \alpha$, is positive, $a_{03}=1$,

$$
\begin{aligned}
a_{11}= & \left(x_{c}^{2} a_{21}+y_{c}^{2}-1\right) / x_{c} \\
b_{02}= & \left(x_{c}^{4} y_{c}^{2}\left(a_{21}^{2}+2 b_{30}\right)+x_{c}^{2} y_{c}^{2}\left(2-y_{c}^{2}\right) a_{21}+\alpha^{2} x_{c}^{2}-2 x_{c}^{2} y_{c}^{2}-y_{c}^{4}+y_{c}^{2}\right) /\left(2 x_{c} y_{c}^{4}\right), \\
b_{12}= & -\left(x_{c}^{4} y_{c}^{2}\left(a_{21}^{2}+2 b_{30}\right)+2 x_{c}^{2} y_{c}^{2} a_{21}+\alpha^{2} x_{c}^{2}-y_{c}^{6}-2 x_{c}^{2} y_{c}^{2}+y_{c}^{2}\right) /\left(2 x_{c}^{2} y_{c}^{4}\right), \\
b_{20}= & \left(x_{c}^{2} y_{c}^{2} a_{21}-2 x_{c}^{4} b_{30}-y_{c}^{4}-2 x_{c}^{2}+y_{c}^{2}\right) /\left(2 x_{c}^{3}\right), \\
\text { and } c_{1}=0 \text { or } c_{2}=0, \text { with } & \\
c_{1}= & x_{c}^{2}\left(x_{c}^{2} a_{21}-y_{c}^{2}+1\right) \alpha^{2}+y_{c}^{2}\left(x_{c}^{6} a_{21}^{3}+3 x_{c}^{4} a_{21}^{2}+2 x_{c}^{6} a_{21} b_{30}\right. \\
& \left.\quad-x_{c}^{2}\left(y_{c}^{4}+2 x_{c}^{2}+y_{c}^{2}-3\right) a_{21}+2 x_{c}^{4}\left(y_{c}^{2}+1\right) b_{30}-2 x_{c}^{2}-y_{c}^{2}+1\right), \\
c_{2}= & x_{c}^{4} y_{c}^{2} a_{21}^{2}-2 x_{c}^{2} y_{c}^{2}\left(y_{c}^{2}-1\right) a_{21}+2 x_{c}^{4} y_{c}^{2} b_{30}+\alpha^{2} x_{c}^{2}-y_{c}^{6}-2 x_{c}^{2} y_{c}^{2}+y_{c}^{2} .
\end{aligned}
$$

Moreover, system (10) either has a polynomial inverse integrating factor when $c_{1}=0$ or a polynomial first integral of degree four when $c_{2}=0$. 
Proof. Straightforward computations show that when $a_{03}=0$ it is not possible to have a weak focus at $\left(x_{c}, y_{c}\right)$. Hence, after a re-scaling of the $(x, y)$ variables if necessary, we have $a_{03}=1$. Then, imposing that system (10) has a non-degenerate weak focus at $\left(x_{c}, y_{c}\right)$, the determinant of the Jacobian matrix at this point, that we call $\alpha$, must be positive and, furthermore, we get conditions (11).

Following the procedure described in Section 3, the first Lyapunov quantity is

$$
L_{1}=-c_{1} c_{2} /\left(4 \alpha^{3} x_{c}^{5} y_{c}^{5}\right),
$$

where $c_{1}$ and $c_{2}$ are given by expressions (12) and (13), respectively. Hence, conditions $c_{1}=0$ or $c_{2}=0$ are required for the existence of a center at $\left(x_{c}, y_{c}\right)$.

When $c_{1}=0$, system (10) has the polynomial inverse integrating factor

$$
\begin{aligned}
V(x, y)= & g_{1} g_{2} x^{4}+g_{1} g_{3} x_{c}^{2} x^{2} y^{2}+2 g_{1} g_{4} x_{c}^{4} y_{c}^{2} y^{4}-g_{1} g_{5} x_{c} x^{3}+2 g_{1} g_{6} x_{c}^{3} x y^{2} \\
& +g_{7} x_{c}^{2} x^{2}+g_{8} x_{c}^{4} y^{2}-g_{4} g_{9} g_{10} x_{c}^{3} y_{c}^{2} x-g_{4}^{2} g_{9} x_{c}^{4} y_{c}^{4}
\end{aligned}
$$

where

$$
\begin{aligned}
g_{1}= & \left(x_{c}^{2} a_{21}+y_{c}^{2}-1\right) x_{c}^{2} \alpha^{2}-y_{c}^{2}\left(x_{c}^{2} a_{21}-2 x_{c}^{2}-y_{c}^{2}+1\right), \\
g_{2}= & x_{c}^{2}\left(x_{c}^{2} a_{21}-y_{c}^{2}+1\right) \alpha^{2}+y_{c}^{2}\left(x_{c}^{6} a_{21}^{3}+3 x_{c}^{4} a_{21}^{2}-\left(y_{c}^{4}+2 x_{c}^{2}+y_{c}^{2}-3\right) x_{c}^{2} a_{21}\right. \\
& \left.-2 x_{c}^{2}-y_{c}^{2}+1\right) \\
g_{3}= & 2 \alpha^{2} x_{c}^{2}+y_{c}^{2}\left(3 x_{c}^{4} a_{21}^{2}+x_{c}^{2}\left(2 y_{c}^{2}+5\right) a_{21}-y_{c}^{4}-2 x_{c}^{2}-y_{c}^{2}+2\right), \\
g_{4}= & x_{c}^{2} a_{21}+y_{c}^{2}+1 \\
g_{5}= & 2 x_{c}^{2}\left(x_{c}^{2} a_{21}-y_{c}^{2}+1\right) \alpha^{2}+y_{c}^{2}\left(x_{c}^{6} a_{21}^{3}+\left(y_{c}^{2}+4\right) x_{c}^{4} a_{21}^{2}-x_{c}^{2}\left(y_{c}^{4}+6 x_{c}^{2}-5\right) a_{21}\right. \\
& \left.-y_{c}^{6}-2 x_{c}^{2} y_{c}^{2}-6 x_{c}^{2}-y_{c}^{2}+2\right), \\
g_{6}= & 2 \alpha^{2} x_{c}^{2}+y_{c}^{2}\left(x_{c}^{4} a_{21}^{2}+x_{c}^{2}\left(2 y_{c}^{2}+1\right) a_{21}+y_{c}^{4}-2 x_{c}^{2}-y_{c}^{2}\right), \\
g_{7}= & -2 x_{c}^{4}\left(x_{c}^{2} a_{21}-y_{c}^{2}+1\right) \alpha^{4}+x_{c}^{2} y_{c}^{2}\left(x_{c}^{6} a_{21}^{3}-x_{c}^{4}\left(4 x_{c}^{2}-y_{c}^{2}+2\right) a_{21}^{2}-4 x_{c}^{2} y_{c}^{4}-y_{c}^{6}\right. \\
& \left.-x_{c}^{2}\left(8 x_{c}^{2} y_{c}^{2}+y_{c}^{4}-6 x_{c}^{2}-6 y_{c}^{2}+7\right) a_{21}-2 x_{c}^{2} y_{c}^{2}+10 x_{c}^{2}+5 y_{c}^{2}-4\right) \alpha^{2} \\
& +\left(x_{c}^{2} a_{21}-2 x_{c}^{2}-y_{c}^{2}+1\right)\left(x_{c}^{6} a_{21}^{3}+2 x_{c}^{4} y_{c}^{2} a_{21}^{2}+x_{c}^{2}\left(y_{c}^{4}+6 x_{c}^{2}+3 y_{c}^{2}-3\right) a_{21}\right. \\
& \left.+4 x_{c}^{2} y_{c}^{2}+y_{c}^{4}+6 x_{c}^{2}+y_{c}^{2}-2\right) y_{c}^{4}, \\
g_{8}= & -4 \alpha^{4} x_{c}^{4}-2 x_{c}^{2} y_{c}^{2}\left(x_{c}^{4} a_{21}^{2}+2 x_{c}^{2} y_{c}^{2} a_{21}+2 x_{c}^{2} a_{21}+y_{c}^{4}-4 x_{c}^{2}-2 y_{c}^{2}+1\right) \alpha^{2} \\
& +y_{c}^{4}\left(x_{c}^{2} a_{21}-2 x_{c}^{2}-y_{c}^{2}+1\right)\left(x_{c}^{4} a_{21}^{2}+x_{c}^{2}\left(2 y_{c}^{2}+3\right) a_{21}+y_{c}^{4}+2 x_{c}^{2}+5 y_{c}^{2}+2\right), \\
g_{9}= & x_{c}^{2} a_{21}-2 x_{c}^{2}-y_{c}^{2}+1, \\
g_{10}= & -2 \alpha^{2} x_{c}^{2}+y_{c}^{2}\left(x_{c}^{4} a_{21}^{2}+x_{c}^{2}\left(2 y_{c}^{2}-1\right) a_{21}+y_{c}^{4}+2 x_{c}^{2}+y_{c}^{2}-2\right) .
\end{aligned}
$$

The proof finishes by direct calculations showing that, when $c_{2}=0$, system (10) is a Hamiltonian system with a degree four polynomial first integral.

Remark 4. In the above proof, before obtaining the inverse integrating factor, two more Lyapunov quantities can be computed. But they vanish when $L_{1}=0$. This fact could indicate that only one limit cycle can be obtained around the equilibrium point lying outside the symmetry axis. 
Proposition 5. The cubic system

$$
\begin{aligned}
& x^{\prime}=-\left(256 x^{2}-147 y^{2}-512 x+844\right) y, \\
& y^{\prime}=\left(4 x^{2}-293 y^{2}-8 x\right)(x-1) .
\end{aligned}
$$

has a center at the origin and two symmetric centers at $(1, \pm 2)$. Moreover, there exists a cubic polynomial perturbation such that it unfolds 9 small limit cycles by a simultaneous degenerate Hopf bifurcation in configuration $\langle 1,7,1\rangle$. In detail, after perturbing, 7 limit cycles surround the origin while each one of the 2 other limit cycles surrounds one of the perturbed symmetric centers.

Proof. The first statement follows because system (14) has the next rational first integral

$$
H(x, y)=\frac{\left(61 x^{2}+183 y^{2}-122 x+211\right)^{5}}{\left(1220 x^{2}-14945 y^{2}-2440 x+3376\right)^{4}} .
$$

The second statement follows from considering the perturbation monomials in two steps, first the reversible perturbation monomials with respect to the origin, and then the non reversible ones. We can proceed in this way because both perturbations are independent. Let us expose these arguments in detail.

First, we perturb system (14) only with the cubic reversible perturbation monomials as it is given in expression (8), that is

$$
\begin{aligned}
& x^{\prime}=-\left(256 x^{2}-147 y^{2}-512 x+844\right) y+f_{21} x^{2} y+f_{03} y^{3}+f_{11} x y+f_{01} y, \\
& y^{\prime}=\left(4 x^{2}-293 y^{2}-8 x\right)(x-1)+f_{30} x^{3}+f_{12} x y^{2}+f_{20} x^{2}+f_{02} y^{2}+f_{10} x .
\end{aligned}
$$

We perturb in this way because this perturbation does not break the symmetry at the origin and, hence, we keep the center at that point. Adding the next conditions,

$$
\begin{array}{ll}
f_{10}=f_{30}+8 f_{03}+4 f_{12}, & f_{11}=-2 f_{21}, \\
f_{01}=f_{21}-4 f_{03}, & f_{02}=-f_{12}, \\
f_{20}=-8 f_{03}-2 f_{30}-4 f_{12}, &
\end{array}
$$

the points $(1, \pm 2)$ remain as equilibrium points of system (15) with zero trace. That is, they are either centers or weak foci. Now, we will study the degenerate Hopf bifurcation and the maximum order at the equilibria $(1, \pm 2)$ as weak foci.

Following the notation introduced in (6), the linear part of the first three Lyapunov quantities, after the change of variables

$$
\left\{f_{30}=y_{4}, f_{21}=y_{3}, f_{12}=y_{2}, f_{03}=\frac{19208}{61} y_{1}-\frac{1}{2} y_{2}+\frac{1}{8} y_{4}\right\},
$$

write as

$$
L_{1}^{(1)}=y_{1}, \quad L_{2}^{(1)}=-\frac{3003925}{4148928} y_{1}, \quad L_{3}^{(1)}=\frac{14324144100569}{15300980932608} y_{1} .
$$

Clearly, using these three quantities and after changing the trace, at $(1, \pm 2)$ we can get only one small limit cycle surrounding each equilibrium by using the double symmetric Hopf bifurcation. Moreover, the origin remains as a center. Computing higher order terms, in the Lyapunov Taylor series expansion on the perturbation coefficients, we can get no more limit cycles surrounding these equilibrium points. 
Secondly, to study the Hopf bifurcation at the origin, we consider a cubic non reversible perturbation of system (14), independent with respect to the above one,

$$
\begin{aligned}
& x^{\prime}=-\left(256 x^{2}-147 y^{2}-512 x+844\right) y+e_{30} x^{3}+e_{12} x y^{2}+e_{20} x^{2}+e_{02} y^{2}, \\
& y^{\prime}=\left(4 x^{2}-293 y^{2}-8 x\right)(x-1)+e_{21} x^{2} y+e_{03} y^{3}+e_{11} x y .
\end{aligned}
$$

Then, the linear parts of the first five Lyapunov quantities at the origin, $L_{j}^{(1)}$, $j=1, \ldots, 5$, are linearly independent with respect to the perturbation parameters $\left\{e_{02}, e_{03}, e_{11}, e_{12}, e_{20}\right\}$, because the matrix expressing these linear parts in terms of the perturbation parameters

$$
\left(\begin{array}{ccccc}
\frac{973}{844} & 0 & \frac{2071}{1688} & 2 & 0 \\
-\frac{314714 \cdots 9820271}{195243 \cdots 1648896} & -\frac{66227 \cdots 4599}{9253 \cdots 83360} & -\frac{211540 \cdots 35229}{15619 \cdots 1680} & -\frac{106973 \cdots 61723}{462662 \cdots 1680} & -\frac{984171 \cdots 3183459}{78097 \cdots 55840} \\
-\frac{200346 \cdots 233733021}{111263 \cdots 38484480} & -\frac{62294 \cdots 25129}{26365 \cdots 915840} & -\frac{39622 \cdots 881503}{44505 \cdots 3937920} & -\frac{575998 \cdots 957887}{329571 \cdots 64480} & -\frac{445637 \cdots 9457639}{22252 \cdots 968960} \\
-\frac{378263 \cdots 05108291}{54817 \cdots 827228672} & \frac{9201158864667}{16237522460672} & -\frac{102311 \cdots 7592337}{10963 \cdots 4457344} & -\frac{47561762093925}{32475044921344} & -\frac{94410143272665}{685223 \cdots 03584} \\
\frac{409395075}{1202423168} & \frac{3583821}{2849344} & -\frac{914393915}{4809692672} & -\frac{206403}{1424672} & \frac{269741035}{2404846336}
\end{array}\right)
$$

has determinant different from zero. Then, by naming $L_{j}^{(1)}=x_{j}, j=1, \ldots, 5$ to these linear parts and by adding $\left\{-50723 e_{30}+64370 e_{21}=x_{6}, e_{30}=x_{7}\right\}$, we get that the linear part of the next two Lyapunov quantities are

$$
\begin{aligned}
L_{6}^{(1)}= & -\frac{66694096304772573135207068214846052608276037011880146949968106767}{118456040720121071307810175534967521775273397089522308616122531840} x_{1} \\
& -\frac{986857882481001983077574408738310242905679762830619750163}{2969506583172454313990723467150121014703569791340500746240} x_{2} \\
& -\frac{368672592894986791238956153658592848962867997427877}{1823801029483205625394394663302399350773808685383680} x_{3} \\
& -\frac{137091470959965151517258036957371407797436275}{64007751590654046173238284436782619114217472} x_{4} \\
& +\frac{20142243532513229363034276626368334529}{11232015437703212769893400803269562944} x_{5}, \\
L_{7}^{(1)}= & -\frac{605442557589991878553884569125575916881324522829664632170711489980338597}{337522008889632653804481076799506498365244602356647852921497039363112960} x_{1} \\
& -\frac{4681103927192298270535681990962679610902010473387521657386808069}{3384458306289173465937433586713357765007811345414923103317786624} x_{2} \\
& -\frac{130456147973103575784262647445829365947450177664718011212431}{166292368657657441583480514160406776823399908315068041789440} x_{3} \\
& -\frac{64452346898380758995060611894437235640368695062374433}{14590408235865645003155157306419194806190469483069440} x_{4} \\
& +\frac{234977057189451422295127519143367170772214655}{256031006362616184692953137747130476456869888} x_{5} .
\end{aligned}
$$

Clearly, up to first order perturbation of the Lyapunov quantities and adding the trace parameter, we can only get 5 small limit cycles surrounding the origin, and no more. Considering all together, using only the first order Lyapunov quantities and the trace parameter, we have 7 limit cycles in configuration $\langle 1,5,1\rangle$ for some cubic systems near system (14).

If we want more limit cycles we can compute, up to order three, the first seven Lyapunov quantities. From the above first order study, using the implicit function theorem, up to a change of variables (in the parameters space), if necessary, we can write $L_{j}=x_{j}+O_{K}(x)$ for $j=1, \ldots, 5$ with $x=\left(x_{1}, \ldots, x_{7}\right)$ and $K \geq 2$. Here $O_{K}(x)$ denotes all the terms of degree at least $K$ on the coordinates of $x$. It is clear that, when $L_{j}=0$ for $j=1, \ldots, 5$, we have $L_{6}^{(1)}=L_{7}^{(1)}=0$. But, in this case, we have also 
$L_{6}^{(2)}=L_{7}^{(2)}=0$. Then, up to order two we have no more limit cycles. Computing the third order and evaluating on $L_{1}=\cdots=L_{5}=0$ we have

$$
L_{j}^{(3)}\left(x_{6}, x_{7}\right)=C_{j}^{(3)} x_{6}^{3} p_{2}\left(x_{7} / x_{6}\right), \quad \text { for } j=6,7,
$$

with

$$
p_{2}(\lambda)=3695279045405753944 \lambda^{2}+271388730148261 \lambda+10087769971
$$

and

$$
\begin{aligned}
C_{6}^{(3)} & =\frac{14810743699301227153984257377365779343617}{2167350437458050857073941269092541778503862023955797053468443248160}, \\
C_{7}^{(3)} & =\frac{422529517425810659146496535729952424790939892023}{24702107859473889845193968445764877445317232939145226398071951833940828160} .
\end{aligned}
$$

Finally, as the above homogeneous parts of degree three are also multiple one from the other and since $L_{7}^{(4)}=0$, we need compute up to order five the first seven Lyapunov quantities. If we proceed in this way, doing the same simplification procedure as in the previous steps, we get

$$
L_{7}^{(5)}\left(x_{6}, x_{7}\right)=C_{7}^{(5)} x_{6}^{5} p_{4}\left(x_{7} / x_{6}\right)
$$

with

and

$$
C_{7}^{(5)}=\frac{9}{19645876735529840 \cdots 17973196784896000000}
$$

$$
\begin{aligned}
p_{4}(\lambda)= & 211148735150868711498020 \cdots 214462220974330859217 \lambda^{4} \\
& +153229267494841185603714 \cdots 695050974950207558339 \lambda^{3} \\
& -278673331636981994254986 \cdots 869624591189537981617 \lambda^{2} \\
& -130937529746819089567596 \cdots 578679565217539087419 \lambda \\
& -227414742460144672742349 \cdots 215962576056358409128 .
\end{aligned}
$$

Then, after some necessary arrangements because of the linear dependence of the first order terms, the Lyapunov quantities up to order five can be written as

$$
\begin{aligned}
& L_{1}=x_{1}+O_{6}\left(x_{1}, \ldots, x_{7}\right), \\
& L_{2}=x_{2}+O_{6}\left(x_{1}, \ldots, x_{7}\right), \\
& L_{3}=x_{3}+O_{6}\left(x_{1}, \ldots, x_{7}\right), \\
& L_{4}=x_{4}+O_{6}\left(x_{1}, \ldots, x_{7}\right), \\
& L_{5}=x_{5}+O_{6}\left(x_{1}, \ldots, x_{7}\right), \\
& L_{6}=C_{6}^{(3)} x_{6}^{3} p_{2}\left(x_{7} / x_{6}\right)+O_{6}\left(x_{1}, \ldots, x_{7}\right), \\
& L_{7}=C_{7}^{(5)} x_{6}^{5} p_{4}\left(x_{7} / x_{6}\right)+O_{6}\left(x_{1}, \ldots, x_{7}\right) .
\end{aligned}
$$

The polynomial $p_{2}$ has two simple real zeros, $\lambda_{1}$ and $\lambda_{2}$, and the resultant of $p_{2}$ and $p_{4}$ is different from zero. Hence, for every $x_{6}$ small enough and different from zero, there exist two curves near $x_{1}=x_{2}=\cdots=x_{5}=0$ and $x_{7}=\lambda_{\ell} x_{6}, \ell=1,2$ such that $L_{j}=0, j=1, \ldots, 6$ and $L_{7} \neq 0$. Moreover, over these curves, the intersection of the varieties $L_{j}$ is transversal. Consequently, as in [7], they define two families of weak foci of order seven that unfold, perturbing also the parameter that defines the trace at the origin, seven small limit cycles via a degenerate Hopf bifurcation. Hence, by computing the Lyapunov quantities up to order five in terms of the perturbation parameters, we have proved the existence of cubic polynomial perturbations 
of system (14) unfolding 9 small limit cycles by a simultaneous degenerate Hopf bifurcation in configuration $\langle 1,7,1\rangle$, as we wanted prove.

Finally, we would like to remark that the homogeneous terms of degree 2 and 4 for all the computed Lyapunov quantities corresponding to the origin of system (16) vanish identically. This explains why, in the above computations, only appear the terms of degrees 1, 3 and 5. Additionally, we have not write down all the figures of all the involved integer numbers in the previous expressions because of their size.

Under the assumptions of Proposition 3 there are other cubic systems with the same configuration of two symmetric centers and one more on the symmetry line and exhibiting the same bifurcation phenomenon. All of them with 9 limit cycles, but having different type of first integrals or inverse integrating factors. We show some of them in the next examples skipping the explicit computations.

The cubic system

$$
\begin{aligned}
& x^{\prime}=y^{3}-y, \\
& y^{\prime}=2 x^{3}+\frac{1}{6} x y^{2}-3 x^{2}-\frac{1}{12} y^{2}+x,
\end{aligned}
$$

has the inverse integrating factor

$$
V=\left(12 x^{2}-8 y^{2}-12 x+9\right)\left(12 x^{2}+9 y^{2}-12 x-8\right),
$$

and the polynomial first integral

$$
H=\left(12 x^{2}-8 y^{2}-12 x+9\right)^{9}\left(12 x^{2}+9 y^{2}-12 x-8\right)^{8} .
$$

Not all the systems with the described configuration have a polynomial or rational first integral. For example the cubic system

$$
\begin{aligned}
& x^{\prime}=-6 y x^{2}+y^{3}+3 x y-y, \\
& y^{\prime}=-\frac{3}{4} x y^{2}-\frac{5}{8} x^{3}+\frac{21}{4} y^{2}-\frac{147}{8} x^{2}+x,
\end{aligned}
$$

with an integrating factor

$V=205 x^{4}-1722 x^{2} y^{2}+328 y^{4}+14678 x^{3}-2460 x y^{2}-12050 x^{2}+1612 y^{2}+2376 x-176$

has the next first integral

$$
\begin{aligned}
H= & \log \left(205 x^{4}-1722 y^{2} x^{2}+328 y^{4}+14678 x^{3}-2460 x y^{2}-12050 x^{2}+1612 y^{2}\right. \\
& +2376 x-176)+\frac{54}{\sqrt{401}} \operatorname{arctanh}\left(\frac{\sqrt{401}\left(-1722 x^{2}+656 y^{2}-2460 x+1612\right)}{32882 x^{2}-65764 x+33684}\right) .
\end{aligned}
$$

Inside this cubic family, after perturbation, there are also systems sharing this configuration with polynomial first integral. For example, systems

$$
\begin{aligned}
& x^{\prime}=448 x^{2} y+64 y^{3}-80 x y-y, \\
& y^{\prime}=312 x^{3}-448 x y^{2}+17 x^{2}+40 y^{2}+x,
\end{aligned}
$$

and

have

$$
\begin{aligned}
& x^{\prime}=y^{3}-y, \\
& y^{\prime}=-8 x^{3}-3 x^{2}+x,
\end{aligned}
$$

$$
H=468 x^{4}-1344 x^{2} y^{2}-96 y^{4}+34 x^{3}+240 x y^{2}+3 x^{2}+3 y^{2}
$$


and

$$
H=-8 x^{4}-y^{4}-4 x^{3}+2 x^{2}+2 y^{2}
$$

as first integrals, respectively.

Moreover, all the considered systems have different phase portraits in the Poincaré disk. In Figure 2 we depict all of them. The finite equilibrium points are all of them of center or saddle type, meanwhile at infinity the equilibrium points, when they exist, all are of node or saddle type.

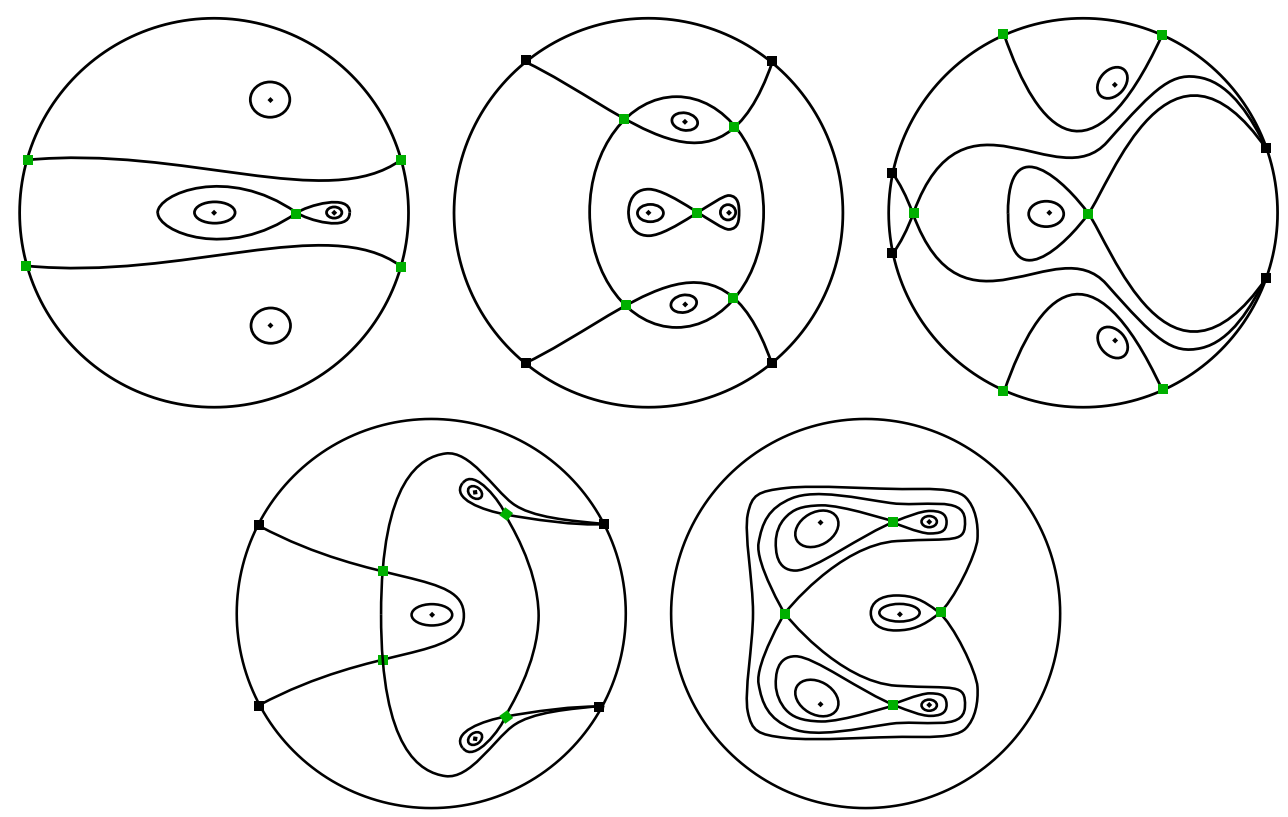

Figure 2. The qualitative phase portraits of cubic systems (14), (17), (18), (19), and (20), on the Poincaré sphere.

Finally, we remark that system (20) belongs to the family studied in [18] where 13 limit cycles appear bifurcating simultaneously from different period annulus.

\section{REVERSIBle QUARTIC SYSTEMS}

Christopher, in [7], study the simultaneous bifurcation of limit cycles in configuration $\langle n, m, n\rangle$ for a quartic Darboux center given by Żołądek in [32]. Using the method described in Section 3, up to first order, i.e. calculating the linear parts of the Lyapunov quantities, he provides 22 limit cycles in configuration $\langle 6,10,6\rangle$.

In this section we extend this study to some reversible quartic systems having rational first integrals, using linear and higher order developments of the Lyapunov quantities. More concretely, let us consider rational first integrals of the type

$$
H_{N, d}(x, y)=\frac{\left(y^{2}+p_{N, d-1}(x)\right)^{d}}{\left(x y^{2}+q_{N, d}(x)\right)^{d-1}} .
$$


The given polynomials $p_{N, d-1}$ and $q_{N, d}$ are of degree $d-1$ and $d$, respectively. The corresponding differential system writes as

$$
\begin{aligned}
x^{\prime} & =-\frac{\partial H_{N, d}}{\partial y} V_{N, d}(x, y), \\
y^{\prime} & =\frac{\partial H_{N, d}}{\partial x} V_{N, d}(x, y),
\end{aligned}
$$

for a suitable inverse integrating factor $V_{N, d}(x, y)$. All the reversible systems presented in this section are symmetric with respect to the $x$-axis having a center at the origin and two more at $\left(x_{c}, \pm y_{c}\right)$. All of them are non degenerate.

In the first subsection, using first order of Lyapunov quantities and simultaneous bifurcations, we obtain different configurations with 16, 19, and 22 limit cycles, increasing with $d$. The last one, $H_{4,5}$, is in the same class as Christopher's center and we recover his result. In the second subsection, using fifth order developments, we get the new lower bound $H(4) \geq 28$, in a simultaneous bifurcation and in a $\langle 8,12,8\rangle$ configuration. See Proposition 6 . As in the cubic family studied in the previous section, only the developments of odd order are useful for obtaining more limit cycles.

5.1. Configurations found in the class $H_{4, d}$. Next table shows the results on simultaneous bifurcation of limit cycles for system (22), of degree four, and $d=$ $3,4,5$, using the linear parts of the Lyapunov quantities. In the second row we indicate, at the top, the found configuration and, at the bottom, the total number of small limit cycles.

\begin{tabular}{|c|c|c|}
\hline$H_{4,3}$ & $H_{4,4}$ & $H_{4,5}$ \\
\hline$\langle 4,8,4\rangle$ & $\langle 5,9,5\rangle$ & $\langle 6,10,6\rangle$ \\
$(16)$ & $(19)$ & $(22)$ \\
\hline
\end{tabular}

Because of the size of the expressions of the Lyapunov quantities and as we are following exactly the procedure described in Section 3 we only list the polynomials that define the rational first integrals (21):

$$
\begin{aligned}
p_{4,2}(x) & =-\frac{24961}{24336} x^{2}+2 x-1, \\
q_{4,3}(x) & =\frac{84551}{36504} x^{3}-3 x^{2}+3 x-1, \\
p_{4,3}(x) & =\frac{2230433}{473616} x^{3}+\frac{259}{198} x^{2}-\frac{67}{66} x-1, \\
q_{4,4}(x) & =\frac{2230433}{631488} x^{4}+\frac{259}{198} x^{3}+\frac{50}{33} x^{2}-\frac{134}{99} x-1, \\
p_{4,4}(x) & =-\frac{3}{4} x^{4}+2 x^{2}+\frac{4}{3} x+\frac{4}{3}, \\
q_{4,5}(x) & =-\frac{3}{5} x^{5}+2 x^{3}+\frac{4}{3} x^{2}+\frac{2}{3} x+\frac{8}{15} .
\end{aligned}
$$

A more detailed study of the last family is done in Proposition 6 below.

We point out that the number of limit cycles strictly increases, with $d$, in each studied example. We have not shown the results done for higher $d$ because they are worse than those presented. 
5.2. Higher order studies for a quartic system. In Proposition 5 we have seen how higher order developments of the Lyapunov quantities can be used to increase the number of small limit cycles. In that case only from the center on the symmetry line we can get more limit cycles, passing from the configuration $\langle 1,5,1\rangle$, with a first order study, to the configuration $\langle 1,7,1\rangle$, with a fifth order development.

In this subsection, we get the new lower bound of $H(4) \geq 28$ by proceeding in a similar manner to the previously exposed. Due to the difficulties on the computations we have only check how the number of limit cycles increases for a system of degree four which is of type $H_{4,5}$. As we have mentioned above, we have selected a Darboux center which is in the same class as the one studied by Christopher.

Proposition 6. The quartic differential system associated to the rational first integral

$$
H(x, y)=\frac{\left(2 x^{4}-x^{2}+y^{2}-2 x-2\right)^{5}}{\left(8 x^{5}-5 x^{3}+5 x y^{2}-10 x^{2}-5 x-4\right)^{4}}
$$

has one reversible center at the origin and two more centers at the points $(1, \pm 2)$. Perturbing with polynomials of degree four, from these centers bifurcate 22,27 , and 28 limit cycles in configuration $\langle 6,10,6\rangle,\langle 8,11,8\rangle$, and $\langle 8,12,8\rangle$, using the series expansion of the Lyapunov quantities on the perturbation coefficients up to order 1 , 3 , and 5 , respectively.

Proof. It is easy to check that the reversible differential equation associated to the rational first integral $(23)$ has a center at $(0,0)$, on the symmetry axis which is the straight line $\{y=0\}$, and two more symmetric centers at $(1, \pm 2)$.

The limit cycles appear via a simultaneous degenerate Hopf bifurcation, following the procedure described in Section 3. More concretely, let us denote by $\tilde{L}_{j}^{(\ell)}$ and $\hat{L}_{j}^{(\ell)}$ the Taylor series expansion of order $\ell$ of the $j$ Lyapunov quantities at the equilibrium points obtained perturbing the differential equation, i.e. near the centers at $(0,0)$ and $(1, \pm 2)$, respectively. We notice that, by the symmetric perturbation procedure, the $j$ Lyapunov quantities at $(1,2)$ and $(1,-2)$ coincide.

We will follow the same scheme described in the proof of Proposition 5.

Straightforward computations provide, after some linear changes in the parameters space, the first Lyapunov quantities up to order 1 . We can write them as $\tilde{L}_{j}^{(1)}=x_{j}+O_{2}(x)$, for $j=1, \ldots, 10$ and $\hat{L}_{j}^{(1)}=y_{j}+O_{2}(y)$, for $j=1, \ldots, 6$. We have denoted by $\mathrm{O}_{2}(x)$ and $\mathrm{O}_{2}(y)$ all the terms of degree two in variables $x_{j}$ and $y_{j}$, respectively. Moreover, we observe that $\tilde{L}_{11}^{(1)}, \tilde{L}_{12}^{(1)}, \hat{L}_{7}^{(1)}$, and $\hat{L}_{8}^{(1)}$ are linear combinations of the first ten and six Lyapunov quantities up to order 1, respectively. From these expressions, adding the perturbation parameters that control the traces at the critical points after the perturbation, we get the same configuration of limit cycles, $\langle 6,10,6\rangle$, given by Christopher in [7]. For obtaining more limit cycles, before using the "trace parameters", we need to compute the Taylor series expansion of higher order of the next Lyapunov quantities, $\tilde{L}_{j}$, for $j=11,12$, and $\hat{L}_{j}$, for $j=7,8$.

The second order terms in the Taylor developments of the Lyapunov quantities are zero when the parameters that control first order developments vanish, i.e. when $x_{1}=\cdots=x_{10}=y_{1}=\cdots=y_{6}=0$. Then, third order of Taylor developments of the Lyapunov quantities are necessary to be computed so that more limit cycles could 
be obtained. If we compute them, we obtain the expressions

$$
\begin{aligned}
& \tilde{L}_{11}^{(3)}=C_{1} x_{11} x_{12}^{2} p_{2}\left(x_{11} / x_{12}\right), \\
& \tilde{L}_{12}^{(3)}=C_{2} x_{11} x_{12}^{2} p_{2}\left(x_{11} / x_{12}\right), \\
& \hat{L}_{7}^{(3)}=C_{3} y_{8}^{3} p_{3}\left(y_{7} / y_{8}\right), \\
& \hat{L}_{8}^{(3)}=C_{4} y_{8}^{3} \hat{p}_{3}\left(y_{7} / y_{8}\right),
\end{aligned}
$$

for some constants $C_{1}, C_{2}, C_{3}$, and $C_{4}$ and polynomials

$$
\begin{aligned}
p_{2}(\lambda)= & 837649339589516+3741225987316 \lambda+431335779 \lambda^{2}, \\
p_{3}(\lambda)= & 20932383446945199488077206112653 \\
& +42671630228676522212733338314426 \lambda \\
& +27681018968522683495059877708953 \lambda^{2} \\
& +5653958549111204034021495620096 \lambda^{3} \\
\hat{p}_{3}(\lambda)= & 46246307507579267760260412353597030559 \\
& +94264434217259234279967744936711259963 \lambda \\
& +61137236767015531358857109782475722749 \lambda^{2} \\
& +12482954391842688462988145099794265513 \lambda^{3} .
\end{aligned}
$$

We want to remark that we have four functions but only three polynomials. From previous expressions, we only have, up to order three, one extra limit cycle surrounding the origin. This is so because $\tilde{L}_{11}^{(3)}$ is different from zero and the polynomial $p_{2}$ appears in the two expressions $\tilde{L}_{11}^{(3)}$ and $\tilde{L}_{12}^{(3)}$. Surrounding each one of the critical points $(1, \pm 2)$ we have two more limit cycles, because the polynomials $p_{3}$ and $\hat{p}_{3}$ are of degree three and they have no common zeros, as it follows from the fact that the resultant of them is not zero. Consequently, up to order three, we have the configuration $\langle 8,11,8\rangle$ of limit cycles.

Finally, the development up to order four of $\tilde{L}_{11}$ and $\tilde{L}_{12}$ coincides but not the corresponding to order five and it writes as $\tilde{L}_{12}^{(5)}=C_{5} x_{12} p_{4}\left(x_{11} / x_{12}\right)$ with

$$
\begin{aligned}
p_{4}(\lambda) & =34144153 \cdots 7015797 \lambda^{4}+64857960 \cdots 8874396 \lambda^{3} \\
& +45819614 \cdots 9678048 \lambda^{2}+14251184 \cdots 3436944 \lambda+16440049 \cdots 2184112 .
\end{aligned}
$$

We have not written completely the above polynomial of degree four, because each coefficient is an integer number with more than 200 figures.

Moreover, the resultant of the polynomials $p_{3}$ and $p_{4}$ is non zero, then the existence of an extra hyperbolic limit cycle is guaranteed. Consequently, we get, at least, twenty-eight small limit cycles in configuration $\langle 8,12,8\rangle$. This finishes the proof.

We have not computed higher order Lyapunov quantities to find more limit cycles because to get the maximum, 28 limit cycles, we have exhausted all the perturbation parameters. To get more limit cycles, if they exist, other mechanisms should be applied.

\section{Simultaneous Bifurcation of higher Degree Systems}

This section is devoted to the perturbation of vector field (22), for degrees $N=$ $5, \ldots, 9$. Here we provide the new best lower bounds $H(5) \geq 37$ and $H(6) \geq 53$, 
among others lower bounds. To reach them we use linear developments of Lyapunov quantities, following the way introduced in the previous sections; that is, by the simultaneous bifurcation of limit cycles in differential systems having the rational first integrals (21).

In the next subsections we only provide the polynomials $p_{N, d-1}$ and $q_{N, d}$, all with rational coefficients, and the number of simultaneous limit cycles in configuration $\langle n, m, n\rangle$ for degrees $N=5, \ldots, 9$. Due to the size of the expressions we have only listed the systems and the configurations. As it can be seen, for every degree $N$, the sequence of the number of limit cycles in each configuration is increasing with $d$, up to the maximal value obtained for $d=2 N-3$. We have not shown the configurations for greater values of $d$ because the total number of limit cycles is less than the previous maximal value. Moreover, when we increase the degree $N$ we get better results in next sections using other type of systems. These are the reasons why we stop the computations at these values.

All the systems are chosen so that the involved rational numbers be as small as possible. We observe that the method described in Section 3 needs a normal form transformation where the square root of the determinant of the Jacobian matrix at the equilibrium points appears in the computations. In most of the cases, we achieve that the determinant of the Jacobian matrix at the equilibrium points be a perfect square. This fact simplifies significantly the involved calculations. Alternatively, when a square root of an integer number appears we look for a system that has this number as small as possible.

For the sake of brevity, we omit all the computations because of their size and since they are similar to the ones shown in the previous sections.

6.1. Configurations found in the class $H_{5, d}$. Next table shows the results on simultaneous bifurcation of limit cycles for system (22) of degree five, and $d=4,5,6,7$. The total number of limit cycles obtained for each system is given in parentheses.

\begin{tabular}{|c|c|c|c|}
\hline$H_{5,4}$ & $H_{5,5}$ & $H_{5,6}$ & $H_{5,7}$ \\
\hline$\langle 8,12,8\rangle$ & $\langle 9,13,9\rangle$ & $\langle 10,14,10\rangle$ & $\langle 11,15,11\rangle$ \\
$(28)$ & $(31)$ & $(34)$ & $(37)$ \\
\hline
\end{tabular}

As in the previous degree four case, the sequences of the number of limit cycles in each period annulus also is strictly increasing. Here the polynomials defining the rational first integrals (21) are:

$$
\begin{aligned}
& p_{5,3}(x)=\frac{1431}{286} x^{3}-1, \\
& q_{5,4}(x)=\frac{27617}{6864} x^{4}+\frac{23}{24} x^{3}+\frac{1}{48} x^{2}-1, \\
& p_{5,4}(x)=\frac{316801}{7040} x^{4}-\frac{96}{5} x^{2}-\frac{104}{5} x-1, \\
& q_{5,5}(x)=\frac{316801}{8800} x^{5}-\frac{96}{5} x^{3}+\frac{71}{5} x^{2}-26 x-1, \\
& p_{5,5}(x)=-\frac{512}{205} x^{5}+8 x^{3}-3 x^{2}+\frac{15}{8} x-1, \\
& q_{5,6}(x)=-\frac{256}{123} x^{6}+8 x^{4}-3 x^{3}-\frac{11}{16} x^{2}+\frac{9}{4} x-1, \\
& p_{5,6}(x)=\frac{961}{686} x^{6}+\frac{31}{7} x^{3}-\frac{31}{42} x^{2}+\frac{1}{7} x-1,
\end{aligned}
$$




$$
q_{5,7}(x)=\frac{2883}{2401} x^{7}+\frac{31}{7} x^{4}-\frac{31}{42} x^{3}+\frac{1}{7} x^{2}+\frac{1}{6} x-1 .
$$

6.2. Configurations found in the class $H_{6, d}$. Next table shows the results on simultaneous bifurcation of limit cycles for system (22) of degree six, and $d=$ $6,7,8,9$. As in the previous case, the total number of limit cycles obtained for each system is also given in parentheses.

\begin{tabular}{|c|c|c|c|}
\hline$H_{6,6}$ & $H_{6,7}$ & $H_{6,8}$ & $H_{6,9}$ \\
\hline$\langle 13,18,13\rangle$ & $\langle 14,19,14\rangle$ & $\langle 15,21,15\rangle$ & $\langle 16,21,16\rangle$ \\
$(44)$ & $(47)$ & $(51)$ & $(53)$ \\
\hline
\end{tabular}

In the above sequences we observe that not all of them are strictly increasing in each period annulus. Here the polynomials defining the rational first integrals (21) are:

$$
\begin{aligned}
& p_{6,5}(x)=-\frac{6}{5} x^{5}-54 x^{4}+243 x^{3}-5103 x^{2}+2510 x-1, \\
& q_{6,6}(x)=-x^{6}-54 x^{5}-\frac{9921}{2} x^{2}+3012 x-1, \\
& p_{6,6}(x)=-\frac{1232}{577} x^{6}+\frac{1232}{577} x^{4}-\frac{3152}{577} x^{3}+\frac{1822}{577} x^{2}+\frac{1232}{577} x+\frac{1232}{577}, \\
& q_{6,7}(x)=-\frac{1056}{577} x^{7}+\frac{1232}{577} x^{5}-\frac{3152}{577} x^{4}+\frac{5158}{1731} x^{3}+\frac{5272}{1731} x^{2}+\frac{1294}{1731} x+\frac{2588}{4039}, \\
& p_{6,7}(x)=\frac{13}{7} x^{7}+4 x^{4}+2 x^{3}-\frac{261}{104} x^{2}-\frac{35}{104} x-1, \\
& q_{6,8}(x)=\frac{13}{8} x^{8}+4 x^{5}+2 x^{4}-\frac{261}{104} x^{3}+\frac{29}{104} x^{2}-\frac{5}{13} x-1, \\
& p_{6,8}(x)=\frac{25}{8} x^{8}+10 x^{4}-\frac{40}{9} x^{3}-\frac{32}{9} x^{2}, \\
& q_{6,9}(x)=\frac{25}{9} x^{9}+10 x^{5}-\frac{40}{9} x^{4}-\frac{32}{9} x^{3}+2 x-\frac{16}{9} .
\end{aligned}
$$

6.3. Configurations found in the class $H_{7, d}$. Next table shows the results on simultaneous bifurcation, and total number in parentheses, of limit cycles for system (22) of degree seven, and $d=8,9,10,11$.

\begin{tabular}{|c|c|c|c|}
\hline$H_{7,8}$ & $H_{7,9}$ & $H_{7,10}$ & $H_{7,11}$ \\
\hline$\langle 19,25,19\rangle$ & $\langle 20,25,20\rangle$ & $\langle 21,26,21\rangle$ & $\langle 22,27,22\rangle$ \\
$(63)$ & $(65)$ & $(68)$ & $(71)$ \\
\hline
\end{tabular}

Besides the above sequences are increasing but not strictly, they are less than expected values comparing with the previous degrees. This fact could be indicate that, for higher values of $N$, the proposed rational first integral (21) will not allow us to improve our results. Here the polynomials $p_{7, d-1}, q_{7, d}$ in (21) are:

$$
\begin{aligned}
& p_{7,7}(x)=\frac{32}{7} x^{7}-8 x^{5}+10 x^{3}+10 x^{2}-16 x+\frac{32}{7}, \\
& q_{7,8}(x)=4 x^{8}-8 x^{6}+11 x^{4}+10 x^{3}-18 x^{2}+8 x-2, \\
& p_{7,8}(x)=-\frac{9}{4} x^{8}-2 x^{5}+12 x^{4}-3 x^{3}-\frac{16}{3} x^{2}+\frac{13}{3}, \\
& q_{7,9}(x)=-2 x^{9}-2 x^{6}+12 x^{5}-3 x^{4}-\frac{49}{9} x^{3}+\frac{4}{3} x^{2}+\frac{26}{9}, \\
& p_{7,9}(x)=-\frac{10}{9} x^{9}+4 x^{5}-2 x^{4}-\frac{3}{10} x^{3}+\frac{151}{90} x^{2}+\frac{16}{9} x-\frac{16}{45},
\end{aligned}
$$




$$
\begin{aligned}
& q_{7,10}(x)=-x^{10}+4 x^{6}-2 x^{5}-\frac{3}{10} x^{4}+\frac{151}{90} x^{3}+\frac{44}{45} x^{2}+\frac{4}{9} x-\frac{2}{25}, \\
& p_{7,10}(x)=-\frac{5}{8} x^{10}-\frac{5}{2} x^{5}+\frac{25}{22} x^{4}+\frac{125}{484} x^{3}-\frac{3337}{484} x^{2}+10 x+\frac{5}{2}, \\
& q_{7,11}(x)=-\frac{25}{44} x^{11}-\frac{5}{2} x^{6}+\frac{25}{22} x^{5}+\frac{125}{484} x^{4}-\frac{3337}{484} x^{3}+10 x^{2}+2 x+\frac{5}{11} .
\end{aligned}
$$

6.4. Configurations found in the classes $H_{8,13}$ and $H_{9,15}$. Next table shows the results on simultaneous bifurcation of limit cycles for system (22), for degree $N=8$ and $d=13$, and degree $N=9$ and $d=15$. The total number of limit cycles obtained for each system is given in parentheses.

\begin{tabular}{|c|c|}
\hline$H_{8,13}$ & $H_{9,15}$ \\
\hline$\langle 28,34,28\rangle$ & $\langle 35,41,35\rangle$ \\
$(8,90)$ & $(9,111)$ \\
\hline
\end{tabular}

Here the polynomials defining the rational first integrals (21) are:

$$
\begin{aligned}
p_{8,12}(x)= & \frac{13}{2} x^{12}+13 x^{6}-6 x^{5}-\frac{18}{13} x^{4}-\frac{108}{169} x^{3}-\frac{2305}{2028} x^{2}+\frac{1}{13} x-1, \\
q_{8,13}(x)= & 6 x^{13}+13 x^{7}-6 x^{6}-\frac{18}{13} x^{5}-\frac{108}{169} x^{4}-\frac{2305}{2028} x^{3}+\frac{1}{13} x^{2}+\frac{1}{12} x-1, \\
p_{9,14}(x)= & -\frac{583200}{34969} x^{14}+\frac{16200}{187} x^{7}-\frac{7560}{187} x^{6}-\frac{1764}{187} x^{5}-\frac{4116 x^{4}}{935}-\frac{2401}{935} x^{3} \\
& -\frac{109}{5} x^{2}+26 x-\frac{195}{14}, \\
q_{9,15}(x)= & -\frac{544320}{34969} x^{15}+\frac{16200}{187} x^{8}-\frac{7560}{187} x^{7}-\frac{1764}{187} x^{6}-\frac{4116}{935} x^{5}-\frac{2401}{935} x^{4} \\
& -\frac{109}{5} x^{3}+26 x^{2}-30 x+15 .
\end{aligned}
$$

For these degrees we only consider the highest value $d=2 N-3$ because looking at the sequences of degrees 6 and 7 and comparing with degrees 4 and 5, the observed values for the number of limit cycles are less than the expected ones. In next section we present better results for systems with these degrees.

\section{Systems having an Algebraic CURVe of EqUilibria}

In this section, we consider the system (22) adding curves of equilibrium points. Of course, the first integrals are the same but the degree is increased. We will see that, for some degrees, this approach provides better Hilbert numbers than the ones obtained in the previous section. The degrees considered are $N=5, \ldots, 10$. More concretely, we consider the unperturbed system

$$
\begin{aligned}
x^{\prime} & =-\frac{\partial H_{N, d}}{\partial y} V_{N, d}(x, y) F_{M}(x, y), \\
y^{\prime} & =\frac{\partial H_{N, d}}{\partial x} V_{N, d}(x, y) F_{M}(x, y),
\end{aligned}
$$

where $H_{N, d}$ is defined in (21), $V_{N, d}$ is a given polynomial and the polynomials $F_{M}$, for $M=1,2,3,4$, are

$$
\begin{array}{ll}
F_{1}(x, y)=1-2 x, & F_{3}(x, y)=1-2 x-2 x y^{2}, \\
F_{2}(x, y)=1-2 x-2 y^{2}, & F_{4}(x, y)=1-2 x-2 x y^{2}-3 x^{2} y^{2} .
\end{array}
$$


In this section we provide the new best lower bounds: $H(7) \geq 74, H(8) \geq 96$, $H(9) \geq 120$, and $H(10) \geq 142$.

The number and configurations of limit cycles obtained, using the bifurcation mechanism described in Section 3, are given in the next table. We remark that, as in the previous section, we get the Lyapunov quantities only up to first order.

\begin{tabular}{|cc|c|c|c|c|}
\hline & & $F_{1}$ & $F_{2}$ & $F_{3}$ & $F_{4}$ \\
\hline$H_{4,5}$ & $\langle 6,10,6\rangle$ & $\langle 10,15,10\rangle$ & $\langle 16,21,16\rangle$ & $\langle 22,27,22\rangle$ & $\langle 28,34,28\rangle$ \\
& $(4,22)$ & $(5,35)$ & $(6,53)$ & $(7,71)$ & $(8,90)$ \\
\hline$H_{5,7}$ & $\langle 11,15,11\rangle$ & $\langle 15,21,15\rangle$ & $\langle 23,28,23\rangle$ & $\langle 29,35,29\rangle$ & $\langle 36,41,36\rangle$ \\
& $(5,37)$ & $(6,51)$ & $(7,74)$ & $(8,93)$ & $(9,113)$ \\
\hline$H_{6,9}$ & $\langle 16,21,16\rangle$ & $\langle 21,28,21\rangle$ & $\langle 30,36,30\rangle$ & $\langle 37,43,37\rangle$ & $\langle 45,52,45\rangle$ \\
& $(6,53)$ & $(7,70)$ & $(8,96)$ & $(9,117)$ & $(10,142)$ \\
\hline$H_{7,11}$ & $\langle 22,27,22\rangle$ & $\langle 28,35,28\rangle$ & $\langle 38,44,38\rangle$ & $\langle 45,52,45\rangle$ & \\
& $(7,71)$ & $(8,91)$ & $(9,120)$ & $(10,142)$ & \\
\hline
\end{tabular}

The first column shows the results obtained in the previous section. The rest of the columns display the results obtained for system (24), according $F_{M}$. In each cell of the table we have indicated the limit cycles configuration (at the top) and the degree of the system and the total number of small limit cycles (at the bottom in parentheses).

For every degree $N$, we observe that, in almost all cases, the biggest values of the lower bounds for the Hilbert numbers have been obtained perturbing a good Darboux system giving a vector field of degree $N$, instead of choosing other Darboux systems with lower degree multiplied by a line of equilibria. This is the case for degree 5 and 6.

\section{LOWER BOUNDS FOR THE HILBERT NUMBERS USING DOUBLE SYMMETRY}

This section is devoted to prove Corollary 2. Its proof follows part of the approach introduced in [8] to get an asymptotic lower bound of the form $H(N)>C N^{2} \log N$, for some constant $C$. Even though there are lower bounds that asymptotically behave like this one, and that we do not improve, they do not give better results for small values of $N$, see for instance [14].

Let us consider a polynomial system with $c$ limit cycles. It is not restrictive to assume that all of them are located at the first quadrant, by performing a translation if necessary. Assuming that the polynomial degree $N$ differential system, $\left(x^{\prime}, y^{\prime}\right)=$ $(f(x, y), g(x, y))$, satisfies these hypotheses, the change of variables $(u, v)=\left(x^{2}, y^{2}\right)$ transforms this system to another one of degree $2 N+1$ with $4 c$ limit cycles. Using this method for $N=2,3,4$ we get the values $H(5) \geq 16, H(7) \geq 52$, and $H(9) \geq 112$. These lower bounds are smaller than the ones obtained in the previous sections, in configuration $\langle n, m, n\rangle$. Nevertheless, using this algorithm in a recursive way from the new lower bounds given in Theorem 1, we can easily improve some known results on the Hilbert numbers. Using the new values for $N=6,7,8,9,10$, we get $H(13) \geq 212, H(15) \geq 296, H(17) \geq 384, H(19) \geq 480$, and $H(21) \geq 568$, in a first step, and $H(27) \geq 848, H(31) \geq 1184, H(35) \geq 1536, H(39) \geq 1920$, and $H(43) \geq 2272$, in a second step. But not all these values provide better lower bounds, comparing with the results in [14]. The new ones are the detailed in the first statement of the corollary. 
Following the algorithm explained above, in a recursive way, Gasull, in [10], provides a lower bound for the Hilbert number of the form $H(N)>K_{N} N^{2}$, where $K_{N}=$ $c_{N} /\left(N^{2}+1\right)^{2}$ and $c_{N}$ is the number of limit cycles of the starting system. Hence, the second statement of the corollary follows using the results presented in this work for $N=2, \ldots, 10$. We remark that for every $c_{N}$ in $4,13,28,37,53,74,96,120,142$, the corresponding $K_{N}$ value is

$$
\frac{4}{9}, \frac{13}{16}, \frac{28}{25}, \frac{37}{36}, \frac{53}{49}, \frac{37}{32}, \frac{32}{27}, \frac{6}{5}, \frac{142}{121},
$$

being $6 / 5$ the biggest one.

\section{AcKnowledgements}

This work has been realized thanks to the MINECO grants MTM2013-40998-P, MTM2014-54275-P, MTM2016-77278-P (FEDER), and UNAB13-4E-1604 (FEDER), and the AGAUR grant 2014 SGR568,

\section{REFERENCES}

[1] A. A. Andronov, E. A. Leontovich, I. I. Gordon, and A. G. Maier. Theory of bifurcations of dynamic systems on a plane. Halsted Press [A division of John Wiley \& Sons], New YorkToronto, Ont.; Israel Program for Scientific Translations, Jerusalem-London, 1973. Translated from the Russian.

[2] N. N. Bautin. On the number of limit cycles appearing with variation of the coefficients from an equilibrium state of the type of a focus or a center. Mat. Sbornik N.S., 30(72):181-196, 1952.

[3] T. R. Blows and N. G. Lloyd. The number of limit cycles of certain polynomial differential equations. Proceedings of the Royal Society of Edinburgh: Section A Mathematics, 98(34):215239, 1984.

[4] Y. L. Bondar' and A. P. Sadovskiı̌. On a theorem of Żołądek. Differ. Uravn., 44(2):263-265, $287,2008$.

[5] L. Chen and M. Wang. The relative position and number of limit cycles of the quadratic differential system. Acta Math. Sin., 22:751-758, 1979.

[6] C. Chicone and M. Jacobs. Bifurcation of limit cycles from quadratic isochrones. J. Differential Equations, 91(2):268-326, 1991.

[7] C. Christopher. Estimating limit cycle bifurcations from centers. In Differential equations with symbolic computation, Trends Math., pages 23-35. Birkhäuser, Basel, 2005.

[8] C. J. Christopher and N. G. Lloyd. Polynomial systems: a lower bound for the Hilbert numbers. Proc. Roy. Soc. London Ser. A, 450(1938):219-224, 1995.

[9] F. Dumortier, J. Llibre, and J. C. Artés. Qualitative theory of planar differential systems. Universitext. Springer-Verlag, Berlin, 2006.

[10] A. Gasull. From Abel's differential equations to Hilbert's sixteenth problem. Butl. Soc. Catalana Mat., 28(2):123-146, 233, 2013.

[11] J. Giné. Higher order limit cycle bifurcations from non-degenerate centers. Appl. Math. Comput., 218(17):8853-8860, 2012.

[12] M. Han. Liapunov constants and Hopf cyclicity of Lienard systems. Ann. of Diff. Eqs, 15(2):113-126, 1999.

[13] M. Han. Bifurcation Theory of Limit Cycles. Alpha Science International Limited, 2016.

[14] M. Han and J. Li. Lower bounds for the Hilbert number of polynomial systems. J. Differential Equations, 252(4):3278-3304, 2012.

[15] T. Johnson. A quartic system with twenty-six limit cycles. Exp. Math., 20(3):323-328, 2011.

[16] T. Johnson and W. Tucker. An improved lower bound on the number of limit cycles bifurcating from a Hamiltonian planar vector field of degree 7. Internat. J. Bifur. Chaos Appl. Sci. Engrg., 20(5):1451-1458, 2010. 
[17] T. Johnson and W. Tucker. An improved lower bound on the number of limit cycles bifurcating from a quintic Hamiltonian planar vector field under quintic perturbation. Internat. J. Bifur. Chaos Appl. Sci. Engrg., 20(1):63-70, 2010.

[18] C. Li, C. Liu, and J. Yang. A cubic system with thirteen limit cycles. J. Differential Equations, 246(9):3609-3619, 2009.

[19] J. Li and Y. Liu. New results on the study of $Z_{q}$-equivariant planar polynomial vector fields. Qual. Theory Dyn. Syst., 9(1-2):167-219, 2010.

[20] H. Liang and J. Torregrosa. Parallelization of the Lyapunov constants and cyclicity for centers of planar polynomial vector fields. J. Differential Equations, 259(11):6494-6509, 2015.

[21] P. Mardesic. An explicit bound for the multiplicity of zeros of generic abelian integrals. Nonlinearity, 4(3):845, 1991.

[22] H. Movasati. Center conditions: rigidity of logarithmic differential equations. Journal of Differential Equations, 197(1):197-217, 2004.

[23] R. Roussarie. Bifurcation of planar vector fields and Hilbert's sixteenth problem, volume 164 of Progress in Mathematics. Birkhäuser Verlag, Basel, 1998.

[24] S. L. Shi. A method of constructing cycles without contact around a weak focus. J. Differential Equations, 41(3):301-312, 1981.

[25] X. Sun and M. Han. On the number of limit cycles of a $Z_{4}$-equivariant quintic near-Hamiltonian system. Acta Math. Sin. (Engl. Ser.), 31(11):1805-1824, 2015.

[26] S. Wang and P. Yu. Bifurcation of limit cycles in a quintic Hamiltonian system under a sixth-order perturbation. Chaos Solitons Fractals, 26(5):1317-1335, 2005.

[27] S. Wang and P. Yu. Existence of 121 limit cycles in a perturbed planar polynomial Hamiltonian vector field of degree 11. Chaos Solitons Fractals, 30(3):606-621, 2006.

[28] Y. Wu, Y. Gao, and M. Han. On the number and distributions of limit cycles in a quintic planar vector field. International Journal of Bifurcation and Chaos, 18(07):1939-1955, 2008.

[29] Y. H. Wu, X. D. Wang, and L. X. Tian. Bifurcations of limit cycles in a $Z_{4}$-equivariant quintic planar vector field. Acta Mathematica Sinica, English Series, 26(4):779-798, Apr 2010.

[30] Y. Q. Ye, S. L. Cai, L. S. Chen, K. C. Huang, D. J. Luo, Z. E. Ma, E. N. Wang, M. S. Wang, and X. A. Yang. Theory of limit cycles, volume 66 of Translations of Mathematical Monographs. American Mathematical Society, Providence, RI, second edition, 1986.

[31] H. Żołądek. Eleven small limit cycles in a cubic vector field. Nonlinearity, 8(5):843-860, 1995.

[32] H. Żołądek. Remarks on "The classification of reversible cubic systems with center". Topol. Methods Nonlinear Anal., 8(2):335-342, 1996.

[33] H. Żołądek. The CD45 case revisited. In Mathematical sciences with multidisciplinary applications: in honor of professor Christiane Rousseau. And in recognition of the mathematics for planet earth initiative, pages 595-625, Cham, 2016. Springer International Publishing.

Dept. de Matemàtiques i Informàtica, Escola Politècnica Superior, Universitat de les Illes Balears. 07122, Palma de Mallorca. Spain

E-mail address: rafel.prohens@uib.cat

Dept. de Matemàtiques, Universitat Autònoma de Barcelona, Edifici C. 08193

Bellaterra, Barcelona. Spain

E-mail address: torre@mat.uab.cat 